\title{
Fine Particulate Matter-induced Toxic Effects in an Animal Model of Caenorhabditis elegans
}

\author{
Meng-Ching Chung ${ }^{1}$, Ming-Hsien Tsai ${ }^{2+}$, Danielle E. Que ${ }^{3}$, Sayre J. Bongo ${ }^{4}$, Wen-Li Hsu ${ }^{1,5}$, \\ Lemmuel L. Tayo ${ }^{4}$, Yi-Hsien Lin ${ }^{6}$, Sheng-Lun Lin ${ }^{7,8,9}$, Yan-You Gou ${ }^{1}$, Yi-Chyun Hsu ${ }^{10}$, \\ Wen-Che Hou ${ }^{3}$, Kuo-Lin Huang ${ }^{1+}$, How-Ran Chao ${ }^{1,11+*}$
}

\author{
${ }^{1}$ Emerging Compounds Research Center, Department of Environmental Science and Engineering, College of Engineering, \\ National Pingtung University of Science and Technology, Pingtung 91201, Taiwan \\ ${ }^{2}$ Department of Child Care, College of Humanities and Social Sciences, National Pingtung University of Science and \\ Technology, Pingtung 91201, Taiwan \\ ${ }^{3}$ Department of Environmental Engineering, National Cheng Kung University, Tainan 70101, Taiwan \\ ${ }^{4}$ School of Chemical, Biological and Materials Engineering and Sciences, Mapúa University, Intramuros, Manila 1002, \\ Philippines \\ ${ }^{5}$ Research Institute for Life Support Innovation, Research Organization for Nano and Life Innovation, Waseda University, \\ Shinjuku, Tokyo 162-8480, Japan \\ ${ }^{6}$ Department of Plant Medicine, National Pingtung University of Science and Technology, Pingtung 91201, Taiwan \\ ${ }^{7}$ Department of Civil Engineering and Geomatics, Cheng Shiu University, Kaohsiung 83347, Taiwan \\ ${ }^{8}$ Center for Environmental Toxin and Emerging-Contaminant Research, Cheng Shiu University, Kaohsiung 83347, Taiwan \\ ${ }^{9}$ Super Micro Mass Research and Technology Center, Cheng Shiu University, Kaohsiung 83347, Taiwan \\ ${ }^{10}$ Department of Environmental Engineering, Kun Shan University, Tainan 71003, Taiwan \\ ${ }^{11}$ Institute of Food Safety Management, National Pingtung University of Science and Technology, Pingtung 91201, Taiwan
}

\begin{abstract}
Research has been focused on the health hazards of ambient $\mathrm{PM}_{2.5}$ related to humans. Many $\mathrm{PM}_{2.5}$ toxicity assessments using in vitro studies have focused on $\mathrm{PM}_{2.5}$-bounded hazardous pollutants. However, $\mathrm{PM}_{2.5}$ toxicity assessment by in vivo studies allow for better observation of the overall effects of $\mathrm{PM}_{2.5}$ exposure on entire organisms, making in vivo $\mathrm{PM}_{2.5}$ toxicity assessment relevant. The toxic effects of outdoor $\mathrm{PM}_{2.5}$, collected from National Pingtung University of Science and Technology (NPUST) and Linluo Junior High School (LJHS), Pingtung, Taiwan, on nematode Caenorhabditis

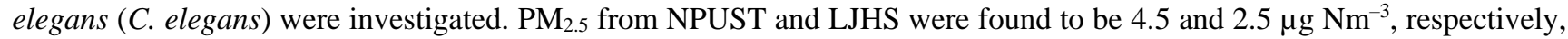
which did not meet the standard. This levels of $\mathrm{PM}_{2.5}$ in Taiwan. For acute toxicity, no significant $\mathrm{PM}_{2.5}$ lethality on C. elegans was observed between NPUST and LJHS. PM 2.5 from NPUST exhibited greater toxicity to lifespan (ageing), locomotion (head thrash), and reproduction (brood size) in the C. elegans animal models than that from LJHS; therefore, adverse effects could be correlated with $\mathrm{PM}_{2.5}$ concentrations. Prolonged exposure to $\mathrm{PM}_{2.5}$ led to more severe toxicity in nematodes as compared to acute exposure. In conclusion, this study suggests that the long-term adverse effects of ambient $\mathrm{PM}_{2.5}$ on environmental organisms should be carefully considered even when $\mathrm{PM}_{2.5}$ is at low levels. C. elegans is a sensitive animal model for the evaluation of $\mathrm{PM}_{2.5}$ ecotoxicity.
\end{abstract}

Keywords: C. elegans; $\mathrm{PM}_{2.5}$; Lifespan; Locomotion; Reproduction; Ageing.

\footnotetext{
+ These authors contributed equally to this work.

* Corresponding author.

Tel.: +886-8-7703202 ext. 7517; Fax: +886-8-7740256

E-mail address: hrchao@mail.npust.edu.tw
}

\section{INTRODUCTION}

Particulate matter $2.5\left(\mathrm{PM}_{2.5}\right)$ is a type of fine particles with an aerodynamic diameter of less than $2.5 \mu \mathrm{m}$. It is produced through processes including natural, indoor, and outdoor combustion processes, as well as other anthropogenic activities (Li et al., 2018a; Martins and da Graça, 2018). Some of the identified major sources of $\mathrm{PM}_{2.5}$ emissions into the atmosphere are power plants (Mari et al., 2016; Dodla et al., 2017), waste incineration facilities (Yan et al., 
2016), and industrial processes, such as the manufacture of steel stacks in the steelmaking industry (Gibson et al., 2013; Owoade et al., 2015). In addition, the large surface area of $\mathrm{PM}_{2.5}$ facilitates easy adhesion of toxic compounds such as polycyclic aromatic hydrocarbons (PAHs), polybrominated diphenyl ethers (PBDEs), polychlorinated biphenyls (PCBs), polychlorinated dibenzo-p-dioxins/furans (PCDD/Fs). on its surface (Pandey et al., 2013; Chao et al., 2016; Lee et al., 2018). The World Health Organization (WHO) established guidelines on air quality that include limitations on exposure to $\mathrm{PM}_{2.5}$ of not more than $25 \mu \mathrm{g} \mathrm{m}^{-3} \mathrm{day}^{-1}$ and $10 \mu \mathrm{g} \mathrm{m}^{-3}$ year $^{-1}$ (Hopke et al., 2018). In Taiwan, the allowable level of exposure to $\mathrm{PM}_{2.5}$

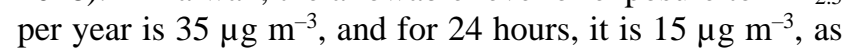
regulated by the Taiwan Air Quality Monitoring Network (TAQMN) of the Environmental Protection Administration, Taiwan (TEPA).

Due to the extremely fine size of $\mathrm{PM}_{2.5}$ particulates, they have been reported to be able to penetrate the blood brain barrier (Feng et al., 2016; Lin et al., 2017). Upon exposure, particulate matter induces overexpression of macrophages in the immune system and oxidative stress resulting in atherosclerotic plaques (Brook and Rajagopalan, 2010) and endothelial and vascular dysfunctions (Feng et al., 2016). $\mathrm{PM}_{2.5}$ also affects the respiratory tract, particularly tiny sacs in the lungs called alveoli, where carbon dioxide and oxygen exchange takes place during respiration (Pinkerton et al., 2000). This can further result in the activation of a series of inflammatory responses in the lungs (Chao et al., 2018; Zhao et al., 2019). $\mathrm{PM}_{2.5}$ is ubiquitous in the environment (Salako et al., 2012; Mohammadyan et al., 2017; Seneviratne et al., 2017), and it has been reported that people suffering from cardiopulmonary and respiratory diseases are at high risk because their exposure to fine particles can cause short-term health effects such as eye, nose, throat and lung irritation, coughing, sneezing, runny nose, and shortness of breath (Kim et al., 2018; Maciejczyk et al., 2018; Polezer et al., 2018). Several in vivo studies utilizing mice models have reported that exposure to airborne $\mathrm{PM}_{2.5}$ prolongs lung tumour progression (Yang and Xiao, 2018), promotes abdominal aortic aneurysms (Jun et al., 2018), increases inflammatory cell infiltration (Hu et al., 2017), and causes lipid accumulation and hepatic function loss (Xu et al., 2019). In vitro studies have associated $\mathrm{PM}_{2.5}$ exposure to induced cytotoxicity and autophagy in human endothelial cells (Zhou et al., 2018), as well as telomere lengthening (Miri et al., 2019). Most importantly, epidemiological studies have shown that both short-term and long-term exposure to PM increases the risk of developing neurological disorders such as stroke (Fu et al., 2019), Parkinson's disease (Hu et al., 2019), and congenital heart disease as a result of prenatal exposure (Huang et al., 2019), and generally stronger associations have been found with mortality and hospitalization (Karimi et al., 2019). Recently, Lo et al. (2017) reported that $\mathrm{PM}_{2.5}$ was involved in deaths resulting from ischemic heart disease, stroke, lung cancer, and chronic obstructive pulmonary disease, wherein a fraction of $18.6 \%$ of the specified diseases was linked to $\mathrm{PM}_{2.5}$ in Taiwan. Therefore, $\mathrm{PM}_{2.5}$ is a major risk factor in Taiwan
(Lung et al., 2016). Currently, there is no known established standard $\mathrm{PM}_{2.5}$ exposure level that may be referenced to avoid its adverse health effects (Kiesewetter et al., 2015). Similarly, there is no singular in vivo animal model that can completely represent the human system. Therefore, there is a need for more research using other available model organisms to further understand the effects of $\mathrm{PM}_{2.5}$ on human health (Feng et al., 2016).

Caenorhabditis elegans (C. elegans) was established by Sydney Brenner as a model organism in 1965 (Brenner, 1974). Compared with single-cell based in vitro assays, $C$. elegans-based assays allow for the evaluation of multiple toxicity endpoints (Kaletta and Hengartner, 2006). At the genetic level, more than half of the conserved signaling regions in humans are found in the nematode (Aoki and Mori, 2015). Numerous studies have reported the sensitivity of C. elegans in terms of pollution from different types of environmental media (Clavijo et al., 2016; Zuo et al., 2017; Rai et al., 2019). However, only a few studies have reported the sensitivity of $C$. elegans to air pollutants, specifically, to $\mathrm{PM}_{2.5}$ (Zhao et al., 2014; Sun et al., 2015, 2016; Wang et al., 2019). In a study by Sun et al. (2015), exposure to coal combustion-related $\mathrm{PM}_{2.5}$ induced lower response in all organism-level endpoints, specifically, prolonged exposure caused more severity than acute exposure to the nematodes. In addition, when Sun et al. (2016) determined the contribution of heavy metals found in coal combustionrelated $\mathrm{PM}_{2.5}$, he found that the combined exposure to specific metals caused greater toxicity than single exposure of said metals. Most importantly, transgenerational effects after exposure to diesel- and traffic-related $\mathrm{PM}_{2.5}$ were discovered by Wang et al. (2019) and Zhao et al. (2014) wherein reproductive disability had been transferred to later generations of $C$. elegans.

C. elegans is an inexpensive, bioethical, and easy model animal to maintain, with attractive physiological characteristics that can be used to assess the toxicological effects of environmental pollutants (Leung et al., 2008). In this study, the toxicity of acute exposure to ambient outdoor $\mathrm{PM}_{2.5}$ samples collected from Southern Taiwanese schools such as National Pingtung University of Science and Technology (NPUST) and Linluo Junior High School (LJHS) was assessed using the different $C$. elegans toxicological endpoints, which include survival (lethality and lifespan), reproduction (brood size), developmental (growth), and locomotion (head thrash and body bend) endpoints.

\section{MATERIALS AND METHODS}

\section{Chemical Reagents}

The wild-type N2 strain of C. elegans was acquired from the Department of Biochemistry and Molecular Biology, College of Medicine, National Cheng Kung University (Tainan, Taiwan) and was maintained in nematode growth medium (NGM) plates containing bacteriological agar and bactopeptone (Laboratories Conda, S.A., Spain) and $\mathrm{NaCl}$ (Honeywell Fluka $^{\mathrm{TM}}$, New Jersey, USA). Additional NGM plate components such as $\mathrm{CaCl}_{2}, \mathrm{~K}_{2} \mathrm{HPO}_{4}$, and cholesterol were obtained from Sigma-Aldrich (St. Louis, MO, USA), 
and $\mathrm{MgSO}_{4}$ was acquired from Avantor Performance Materials, ltd. (Gyeonggi-do, South Korea). OP50 Escherichia coli (E. coli) cultures were acquired from the Bioresources Collection and Research Center (Hsinchu, Taiwan) and Luria-Bertani broth was obtained from SigmaAldrich (St. Louis, MO, USA). For the bleaching solution, $\mathrm{NaOCl}$ was obtained from J.T. Baker (Central Valley, PA), and $\mathrm{KOH}$ was obtained from Duksan Pure Chemicals (Gyeonggi-do, South Korea). $\mathrm{KH}_{2} \mathrm{PO}_{4}$ used for the phosphate buffer was acquired from Avantor Performance Materials, LLC (Radnor, PA, USA), and $\mathrm{Na}_{2} \mathrm{HPO}_{4}$ used for the M9 buffer was obtained from Honeywell Fluka ${ }^{\mathrm{TM}}$ (New Jersey, USA). All physiological observations were done under a dissecting microscope (Olympus, SZX10, Waltham MA, USA). For the K-medium ingredients, $\mathrm{NaCl}$ was obtained from Honeywell Fluka ${ }^{\mathrm{TM}}$ (New Jersey, USA), and $\mathrm{KCl}$ was acquired from Avantor Performance Materials, ltd. (Gyeonggi-do, South Korea). Dehydroethidium (DHE) for oxidative stress detection was purchased from Invitrogen ${ }^{\mathrm{TM}}$, Thermo Fisher Scientific (Waltham, MA USA).

\section{Sample Collection}

Outdoor ambient $\mathrm{PM}_{2.5}$ samples were collected from two southern Taiwanese schools, NPUST and LJHS, located in Neipu, Pingtung County which is in the rural area. The air sampling site in NPUST was set up at the top floor of the College of Engineering in the NPUST campus. The other site in LJHS is nearby Linlou System Interchange of Formosa Highway. Each air sample was collected for 48 hours ( 2 days, $1 \mathrm{~m}^{3} \mathrm{~min}^{-1}$ ) using a high-volume air sampler (SIBATA HV-1000R, Japan) following US EPA Reference Method TO9A. The $\mathrm{PM}_{2.5}$ samples were collected onto quartz fibre filters that were pre-heated before sampling at $600^{\circ} \mathrm{C}$ for 2 hours. A balance with an accuracy of $0.1 \mathrm{mg}$ was used to weigh the filter paper conditioned in an electronic desiccator before and after the sample collection for $24 \mathrm{~h}$. The loaded filters were stored in a refrigerator at $-20^{\circ} \mathrm{C}$ before extraction to limit the possible evaporation of volatile components.

\section{Extraction, Purification, and Sample Preparation}

After the collection of air samples, the mixture was extracted using a Soxhlet extractor with dichloromethane (DCM) for $24 \mathrm{~h}$. The extracts were eluted with $15 \mathrm{~mL}$ DCM during the alumina column clean-up. The eluate was concentrated to approximately $1 \mathrm{~mL}$ and transferred to a vial. The concentrate was further concentrated to near dryness using a stream of nitrogen. For exposure concentrations, $\mathrm{PM}_{2.5}$ was serially diluted $10 \mathrm{X}$ with $1 \%$ DMSO. The toxic effects in the study were limited in the organic fraction.

\section{C. elegans Age Synchronization for Exposure Experiments}

Wild-type N2 C. elegans were maintained on NGM plates seeded with $E$. coli as food and incubated at $22^{\circ} \mathrm{C}$ until the plates had a high density of eggs and gravid nematodes. These were then washed off the plates, placed into centrifuge tubes, and the gravid nematodes were lysed with a bleaching mixture leaving only the eggs to obtain age synchronized populations of L1 nematode larvae in preparation for the exposure experiments and toxicity assays.

\section{Acute Exposure}

Synchronized L1 larvae were seeded onto NGM plates and incubated until L3 or young L4 stage. The L3 and young L4 worms were rinsed from the plate with K-medium, gently washed, and centrifuged at $2500 \mathrm{x}$ g for 4 minutes to remove the $E$. coli in the supernatant by aspirating without disturbing the nematode pellet. After washing, the pellet was re-suspended in $\mathrm{K}$ medium through continuous pipetting, and a seeding volume (worm per $\mu \mathrm{L}$ ) was calculated by pipetting two $5 \mu \mathrm{L}$ drops on a glass slide and manually counting the worms in each drop. Approximately 200 L3/young L4 worms were dispensed into each well of a 12-well plate each containing $1 \mathrm{~mL}$ of the different exposure concentrations for the two $\mathrm{PM}_{2.5}$ samples (one set for NPUST and another for LJHS) diluted with K-medium. Acute (24-hour) exposure was performed at $22^{\circ} \mathrm{C}$ without the presence of food.

\section{Lethality Assay}

The lethality assay was performed by transferring 50 exposed nematodes (per exposure concentration) to NGM plates without food (OP50). These worms were gently prodded using the worm picker to assess viability. Nematodes that were non-responsive to stimulus were scored as dead. The assays were done in triplicates for each of the concentrations.

\section{Lifespan Assay (Ageing Assay)}

Fifty L3/young L4 worms from the acute exposure treatment were transferred to NGM plates with food (OP50). The worms were transferred to fresh NGM plates every day for the first 4 to 5 days (egg-laying period), and after that, the worms were no longer transferred. The plates were incubated at $22^{\circ} \mathrm{C}$. Alive, censored (lost), and dead nematodes were recorded every 2 days within a span of 24 days (general lifespan of C. elegans). The survival plot or the Kaplan-Meier plot was constructed to evaluate the effects of the different concentrations of $\mathrm{PM}_{2.5}$ on the lifespan or ageing of the nematodes. Triplicates were done for each of the exposure concentrations.

\section{Reproductive or Brood Size Assay}

L3/young L4 worms from the acute exposure treatment were assessed for 4 to 5 days of egg-laying. One L3/young L4 worm was transferred to each of the wells of a 12-well NGM plate with a fresh OP50 lawn. Each worm was transferred to a new plate every 2 days within the duration of egg-laying period. The old plates containing the eggs were hatched and incubated to L4 worms for easier counting of progeny. The total progeny for each of the worms was recorded. Twelve worms were evaluated for each of the exposure concentrations.

\section{Growth Measurement Assay}

L3/young L4 worms exposed to each of the $\mathrm{PM}_{2.5}$ concentrations for 24 hours with prolonged exposure were 
measured for their length to assess the effects of $\mathrm{PM}_{2.5}$ on the growth of the nematodes. Ten L3/young L4 nematodes exposed to each of the exposure concentrations were transferred to NGM plates with OP50 lawns and were incubated at $22^{\circ} \mathrm{C}$ for 48 hours until they reached the old L4 stage. The nematodes from each of the concentrations were exposed to a high temperature of $66^{\circ} \mathrm{C}$ in a dry bath for 5 minutes. This process straightens the body of the C. elegans, making it easier to measure the length. An optical microscope (Olympus) was used, and ImageJ software was utilized for the measurement of the worm length.

\section{Locomotion Assay (Head Thrash and Body Bend Assay)}

The endpoints of head thrash and body bending were used to evaluate the locomotion of the exposed L3/young L4 C. elegans by allowing the nematodes swim in K-medium. A head thrash was defined as a change in the direction of the mid body wherein the head and tail falls in the same direction as it moves. Body bending was regarded as a change in direction of the part of the nematodes corresponding to the posterior bulb of the pharynx along the $y$ axis, assuming that the nematode was traveling along the $\mathrm{x}$ axis. Head thrashing was counted for 1 minute, and body bending was counted for 20 seconds. Thirty nematodes were examined per treatment, and three replicates were performed.

\section{Statistical Analysis}

The locomotion, brood size, and length measurement data for the nematodes did not meet the normal distribution using a normality test such as the Shapiro-Wilk test. The Kaplan-Meier plots for the survival rates were used to evaluate the effects of the different $\mathrm{PM}_{2.5}$ concentrations on the lifespan or ageing of the nematodes. The nonparametric Kruskal-Wallis $H$ test was used to examine the differences between each the concentrations in comparison to the control. The survival plots or the Kaplan-Meier plot were derived for the lifespan data using GraphPad Prism 6 (San Diego, California, USA). The significance of each of the days were determined with the Kruskal-Wallis $H$ test. Days with significance were chosen and tested further. Each of the concentrations from the chosen days was also compared to the control using the Mann-Whitney $U$ test. The statistical analyses were tested using SPSS version 12 (International Business Machines Corp., New York, USA).

\section{RESULTS AND DISCUSSION}

\section{Lethality Effects of PM2.5 on C. elegans}

Age-synchronized L4 worms were subjected to 24 hours of exposure to serially diluted samples of $\mathrm{PM}_{2.5}$, after which lethality was investigated. No lethality was observed in any of the $\mathrm{PM}_{2.5}$ sample concentrations of NPUST (0.0014443$\left.1444.3 \mathrm{mg} \mathrm{L}^{-1}\right)$ and LJHS (0.0011518-1151.8 $\left.\mathrm{mg} \mathrm{L}^{-1}\right)$ (Fig. 1) collected from NPUST and LJHS. Similarly, in a study conducted by Sun et al. (2015), coal combustionrelated $\mathrm{PM}_{2.5}$ with exposure concentrations ranging from 0.01 to $100 \mathrm{mg} \mathrm{L}^{-1}$ did not show any lethal effects on nematodes.

\section{Life Span of C. elegans after PM 2.5 Exposure}

In this study, the toxicological endpoint lifespan was also evaluated since exposure of the nematodes to $\mathrm{PM}_{2.5}$ concentrations greater than $1 \mu \mathrm{g} \mathrm{L}^{-1}$ was observed to cause toxicities. The concentrations of $\mathrm{PM}_{2.5}$ determined from NPUST and LJHS were 4.5 and $2.5 \mu \mathrm{g} \mathrm{Nm}^{-3}$ respectively. The detected levels of $\mathrm{PM}_{2.5}$ from both schools were lower than the allowable levels of outdoor $\mathrm{PM}_{2.5}$ in Taiwan, as regulated by the TAQMN. However, prolonged exposure showed that the lifespan of $C$. elegans was significantly reduced for all sample concentrations from the NPUST sampling site. Specifically, the lowest and highest concentrations $\left(1.4443 \times 10^{-3} \mathrm{mg} \mathrm{L}^{-1}\right.$ and $1.4443 \times 10^{3}$ $\mathrm{mg} \mathrm{L}^{-1}$ ) from NPUST caused the sharpest decrease in lifespan starting on post-exposure day 10 (Fig. 2). Additionally, a significant reduction in lifespan was observed on an earlier post-exposure day for LJHS for all concentrations. Concentrations of $1.5518 \times 10^{3}$ and $1.5518 \times 10^{2} \mathrm{mg} \mathrm{L}^{-1}$ caused a rapid decrease in the population of the nematodes.

\section{Acute toxicity}

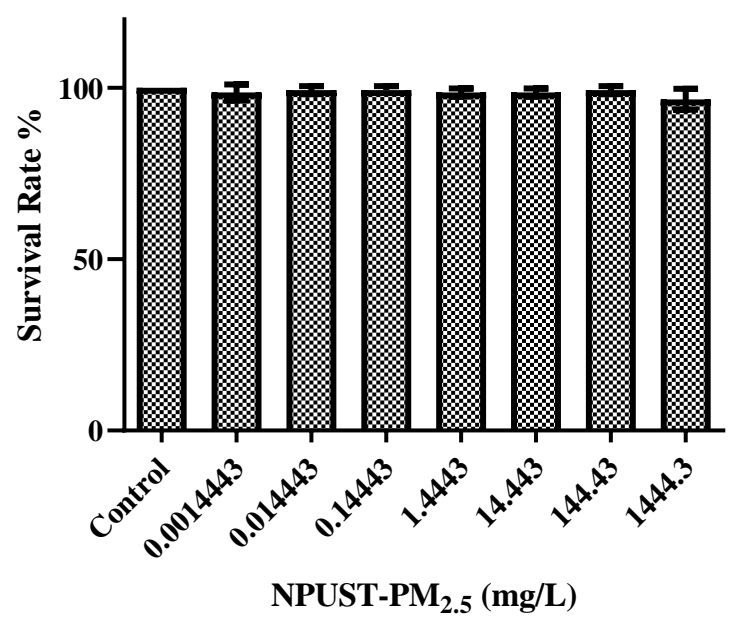

\section{Acute toxicity}

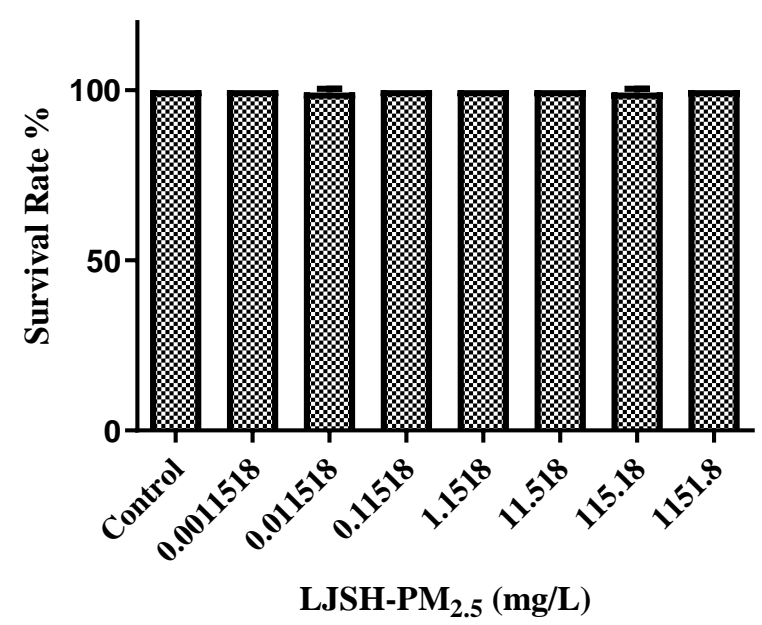

Fig. 1. Dose-dependent lethality of $\mathrm{PM}_{2.5}$ from (Left) NPUST and LJHS after 24-hour exposure. 

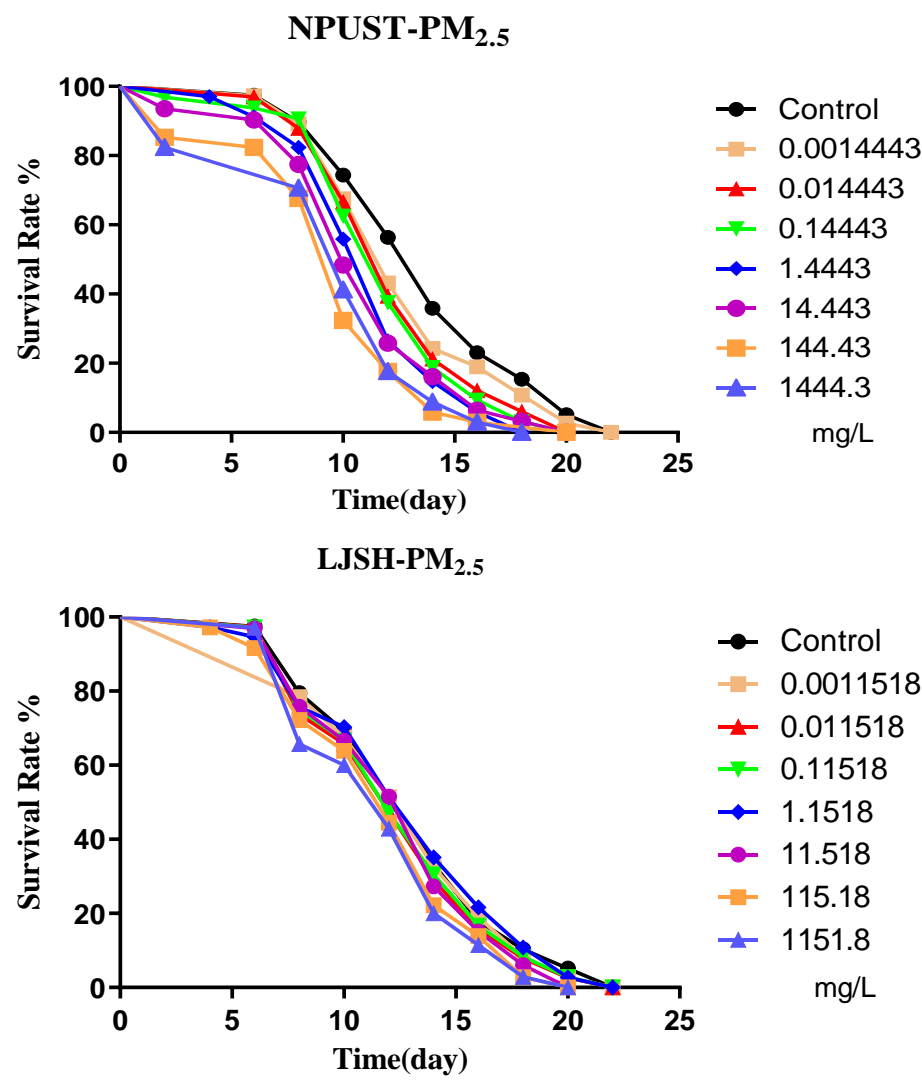

Fig. 2. Effects of $\mathrm{PM}_{2.5}$ from NPUST and (Bottom) LJHS on C. elegans lifespan after 24-hour exposure.

Lastly, C. elegans death occurred on post-exposure day 22, which is two days earlier than the usual lifespan of the nematode. In a similar report by Sun et al. (2015), coal combustion-related $\mathrm{PM}_{2.5}$ at $100 \mathrm{mg} \mathrm{L}^{-1}$ significantly reduced the lifespan of $C$. elegans. However, there was no significant effect on the lifespan when the nematodes were exposed to concentrations $0.01-10 \mathrm{mg} \mathrm{L}^{-1}$, which is inconsistent with the results of this study. Bases on the previous description of the Sun's study (Sun et al., 2015), it was little concern for their study deign. It was difficult to prevent from aggregation of $\mathrm{PM}_{2.5}$ after air sampling, but they didn't show and explain how to maintain $\mathrm{PM}_{2.5}$-type particulate with diversely and homogenously widespread in the nematodes' medium. The finding of our studying is valuable due to our result indicating low levels of $\mathrm{PM}_{2.5}$ exposure in nematodes was still significantly linked into lower lifespan compared with the control. According to the previous reports and the present study (Zhao et al., 2014; Sun et al., 2015, 2016; Wang et al., 2017), $\mathrm{PM}_{2.5}$ exposure decreased lifespan in the in-vivo and epidemiological studies including coal combustion in C. elegans (Sun et al., 2015, 2016), trafficrelated pollutants in the C. elegans (Zhao et al., 2014), ambient air in Drosophila (Wang et al., 2017), ambient air in newborns (Martens et al., 2017), and ambient air in elderly (Li et al., 2018b). Therefore, the level of outdoor air $\mathrm{PM}_{2.5}$ from the two schools, although lower than the national standard in Taiwan, was still shown to have a significant impact on microorganisms and ultimately may pose a threat on human health. To this end, additional endpoints (such as reproductive and locomotion) were investigated to further confirm this effect.

\section{Growth Measurement Assay}

No lethality was observed when the nematodes were subjected to acute exposure with the different concentrations of $\mathrm{PM}_{2.5}$ from either sampling site (NPUST and LJHS). Also, specified concentrations of samples from both the NPUST $\left(1.4443 \times 10^{-3}\right.$ to $\left.1.4443 \times 10^{3} \mathrm{mg} \mathrm{L}^{-1}\right)$ and LJHS $\left(1.5518 \times 10^{-3}\right.$ to $\left.1.5518 \times 10^{3} \mathrm{mg} \mathrm{L}^{-1}\right)$ sampling sites did not lead to the alterations in body length (Fig. 3). Results showed that in all concentrations, the body length of the nematodes remained at $1 \mathrm{~mm}$, the standard length of C. elegans (Leung et al., 2008). A similar study by Sun et al. (2015) reported that acute and prolonged exposure of $C$. elegans to $\mathrm{PM}_{2.5}$ of concentrations 0.01 to $10 \mathrm{mg} \mathrm{L}^{-1}$ had no significant effect on the body lengths of the nematodes under study; however, acute exposure to $\mathrm{PM}_{2.5}$ at a concentration of $100 \mathrm{mg} \mathrm{L}^{-1}$ and prolonged exposure to $\mathrm{PM}_{2.5}$ at concentrations of 10 to $100 \mathrm{mg} \mathrm{L}^{-1}$ significantly reduced body length. Our result was inconsistent with that discussed in Sun's study (Sun et al., 2015).

\section{Effect of Outdoor Air PM2.5 Levels on the Reproductive System of C. elegans}

The secondary targeted organs of environmental pollutants include the neurons and the reproductive system (Leung et al., 2008; Aoki and Mori, 2015). In the C. elegans model, the reproductive system is one of the major targets of 
Growth measurement

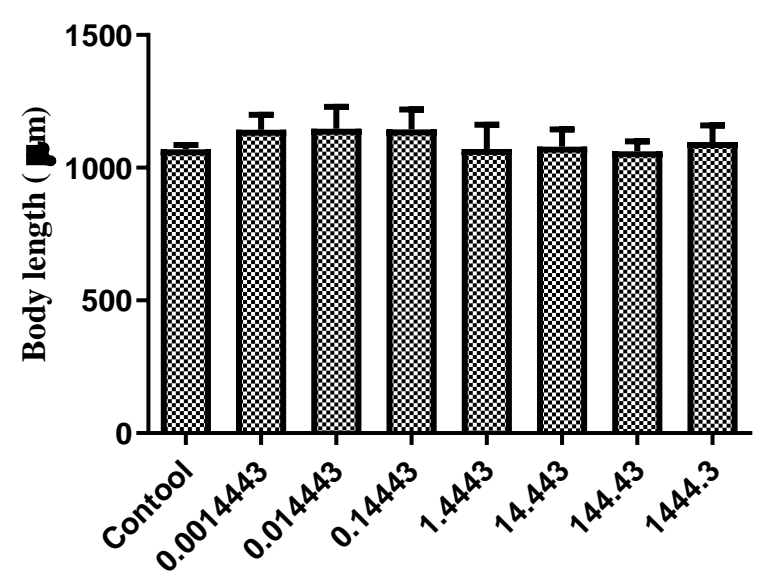

NPUST-PM $2.5(\mathrm{mg} / \mathrm{L})$
Growth measurement

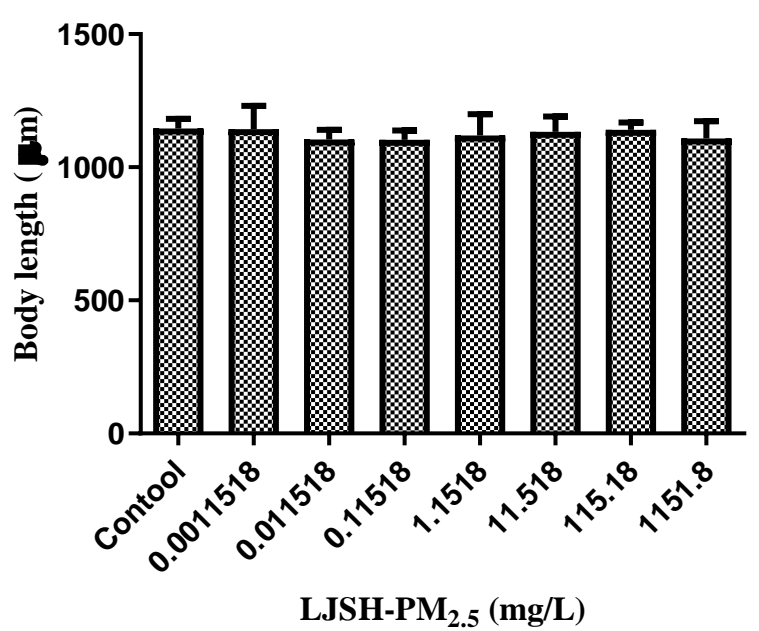

Fig. 3. Effects of $\mathrm{PM}_{2.5}$ from (Left) NPUST and LJHS on C. elegans growth, observed as changes in body length, after 24hour exposure.

toxicants in the body, next to the neurosensory system (Aoki and Mori, 2015). In order to understand the effects of $\mathrm{PM}_{2.5}$ on its translocation in $C$. elegans, the brood size was also assessed for each of the worms exposed to different $\mathrm{PM}_{2.5}$ concentrations (Fig. 4). The total number of eggs, or brood size, was determined twice per concentration of samples from NPUST and LJHS. After acute exposure, the first measurement (Fig. 4(A)) showed that brood size was significantly reduced as the concentration of samples from NPUST increased. Specifically, brood size at 1.4443 $\times 10^{-3} \mathrm{mg} \mathrm{L}^{-1}$ was reduced to 18 eggs laid per worm, and concentrations from $1.4443 \times 10^{-2}$ to $1.4443 \times 10^{3} \mathrm{mg} \mathrm{L}^{-1}$ reduced the brood size to $21,11,10,10,10$, and 3 eggs laid per worm, respectively. Similarly, the second assessment of brood size (Fig. 4(B)) showed that at concentrations ranging from $1.4443 \times 10^{-3}$ to $1.4443 \times 10^{3} \mathrm{mg} \mathrm{L}^{-1}$, the eggs laid per worm decreased to $13,6,5,2,6,3$, and 1 , respectively. For samples gathered from LJHS, the first and second assessment of brood size (Figs. 4(C) and 4(D)) showed no significantly different number of eggs laid per worm at concentrations ranging from $1.5518 \times 10^{-3}$ to $1.5518 \times 10^{3}$ $\mathrm{mg} \mathrm{L}^{-1}$. In this study, the results showed that exposure to high ambient $\mathrm{PM}_{2.5}$ concentrations had a significant effect on the reduction of brood size of $C$. elegans. This result is analogous to the study conducted by Wang et al. (2019), wherein diesel particulate matter (DPM) caused reproduction deficits in C. elegans, as shown by a decrease in brood size during the first generation of offspring. Moreover, in the same study, it was observed that consecutive generation exposure to DPM led to severe limitations in brood size.

Currently, studies have shown that the self-progeny of C. elegans is an effective parameter that can be used to determine the extent of reproductive disability due to the toxicity of various substances (Wang et al., 2018; Moon et $a l ., 2019)$. In general, the effect can be observed in a dosedependent manner (Yang et al., 2018). This was not the case for this study. The control group generated a relatively low brood size of 18 . Dietary restriction (DR) can significantly lower the progeny number in C. elegans (ElHajj and Newman, 2015). In addition, for the size, $\mathrm{PM}_{2.5}$ is greater than E. coli OP50 $(2.5 \mu \mathrm{m}>2 \mu \mathrm{m}$, general food source of C. elegans) (Khan et al., 2018). Therefore, toxicity of $\mathrm{PM}_{2.5}$ is related to the size of the exposed organism.

\section{Effects of Ambient PM2.5 on Locomotion Behavior of C. elegans}

Locomotion assays used to analyze the head thrashing and body bending movements of the nematodes were conducted to assess the neurological toxicity brought about by their exposure to different concentrations of $\mathrm{PM}_{2.5}$ (Fig. 5). A significant reduction in head thrashing movement and body bending were observed for nematodes exposed to $\mathrm{PM}_{2.5}$ sampled from NPUST as compared to nematodes exposed to $\mathrm{PM}_{2.5}$ sampled from LJHS. These results imply that levels of $\mathrm{PM}_{2.5}$ may be correlated to the greatly disrupted functions of the reproductive and neurosensory systems in C. elegans. Additionally, prolonged exposure to $\mathrm{PM}_{2.5}$ was observed to cause more sensitivity to $C$. elegans. Zhao et al. (2014) established that acute exposure to high concentrations of $\mathrm{PM}_{2.5}$ causes adverse effects on both the reproduction and neurosensory functions of $C$. elegans. Moreover, prolonged exposure to low concentrations of $\mathrm{PM}_{2.5}$ further significantly decreased the locomotion behavior of the nematodes. However, this was true for exposure to pollutant concentrations in the range of $\mu \mathrm{g} \mathrm{L}^{-1}$. This is because of the greater dispersion of $\mathrm{PM}_{2.5}$ in DMSO, which increased the bioavailability of the particulate matter to the nematodes (Artifon et al., 2019). The main route of uptake of pollutants in $C$. elegans is through the alimentary system; the worms actively ingest particulates while feeding on bacteria. The presence of bacterial food causes the adsorption of a proportion of the particles and enhances the oral uptake, resulting in higher toxicity (Gonzalez-Moragas et al., 2015). Therefore, the dispersion of particulate matter in the media of choice is important in assessing their effects on in vivo systems. 

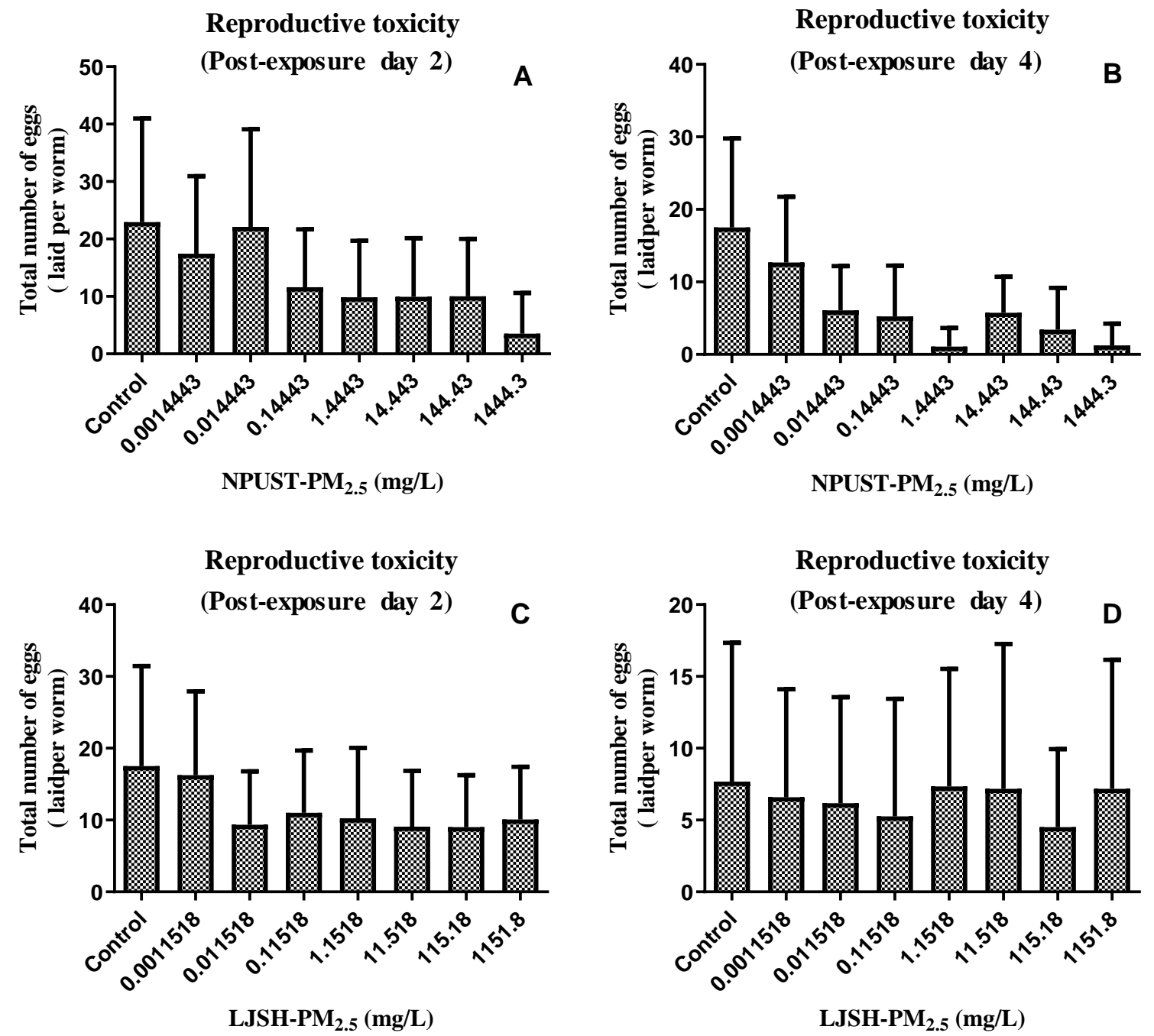

Fig. 4. Effects of $\mathrm{PM}_{2.5}$ from NPUST and (Bottom) LJHS on C. elegans brood size, observed (A and C) two days and (B and D) four days after 24-hour acute exposure.

Several toxic endpoints were examined in the present study. Low $\mathrm{PM}_{2.5}$ levels were found in our sampling areas. To make our amazement, the low levels of $\mathrm{PM}_{2.5}$-bounded pollutants induced several adverse effects including accelerated aging process, reduced reproduction, and delayed locomotion. The present study was limited based on the air sampling technique and pretreatment protocol. The standard methods of $\mathrm{PM}_{2.5}$ air sampling and $\mathrm{PM}$ pretreatment were used in our study. This study excluded in the toxicity in inorganic fraction due to use of the Soxhlet extractors. The present study only considered the toxic effects of $\mathrm{PM}_{2.5}$ bounded hazardous pollutants after the high-volume $\mathrm{PM}_{2.5}$ air samplers mainly due to occurrence of aggregation during the air sampling. Future work will be encouraged to gather airborne $\mathrm{PM}_{2.5}$-type particulate directly into nematodes' medium before aggregation.

\section{CONCLUSIONS}

Low $\mathrm{PM}_{2.5}$ levels were obtained at NPUST and LJHS in this study. It was found that ambient $\mathrm{PM}_{2.5}$ levels at NPUST were higher than the $\mathrm{PM}_{2.5}$ level at LJHS. In addition, the in-vivo model of $C$. elegans was utilized to evaluate the possible health risks associated with differing $\mathrm{PM}_{2.5}$ levels through the investigation of multiple toxic endpoints namely, lethality, lifespan, development, locomotion, and reproduction. The acute toxicity and growth measurement of $C$. elegans was unaffected by $\mathrm{PM}_{2.5}$ collected from NPUST and LJHS, but its lifespan was significantly reduced in the case of airborne $\mathrm{PM}_{2.5}$ at NPUST starting on post-exposure day 10. For the reproductive toxicity and locomotion, reduced brood size and the frequency of head thrashes for NPUST-PM 2.5 was observed, but there were no significant differences in lifespan, brood size, head thrashes, and body bends between the control and LJHS-PM 2.5 . Our significant findings were to indicate the potential toxic effects of long-term exposure to low $\mathrm{PM}_{2.5}$ on $C$. elegans models, even though the measurements were lower than $\mathrm{PM}_{2.5}$ standards. This study implored that the long-term adverse effects of ambient $\mathrm{PM}_{2.5}$ on environmental organisms should be carefully considered even though $\mathrm{PM}_{2.5}$ levels didn't meet the standards. 
Head-thrash frequency

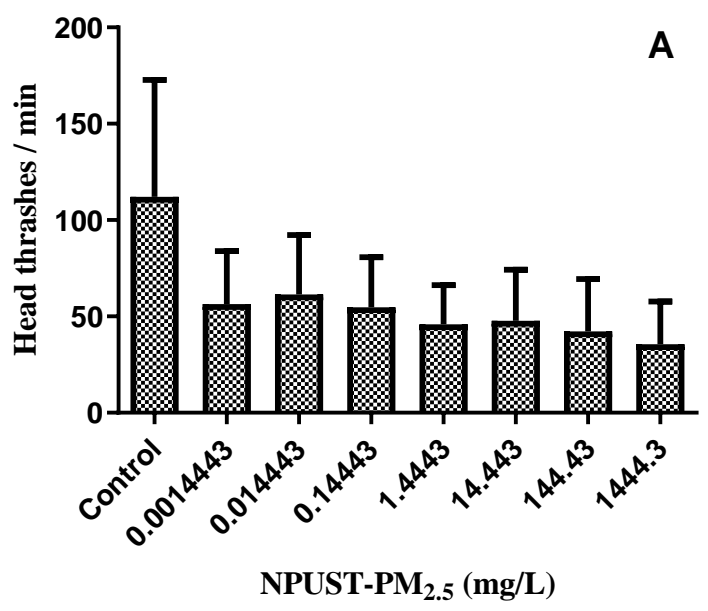

Head-thrash frequency

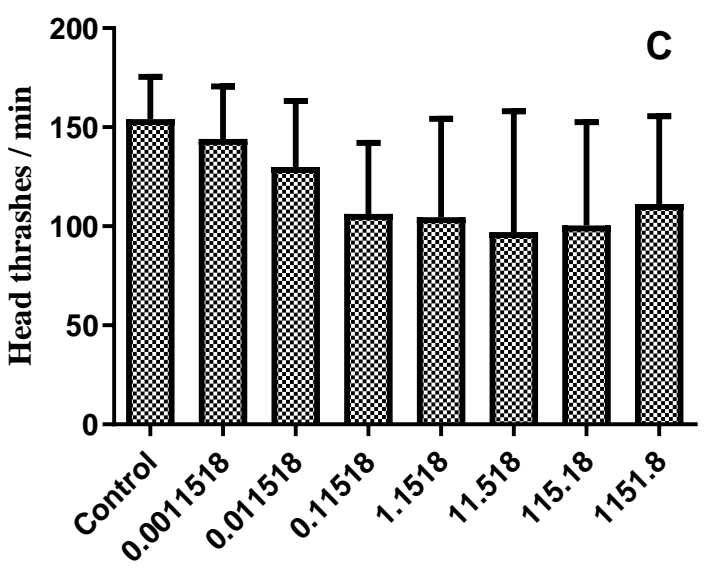

LJSH-PM $2.5(\mathrm{mg} / \mathrm{L})$
Body-bend frequency

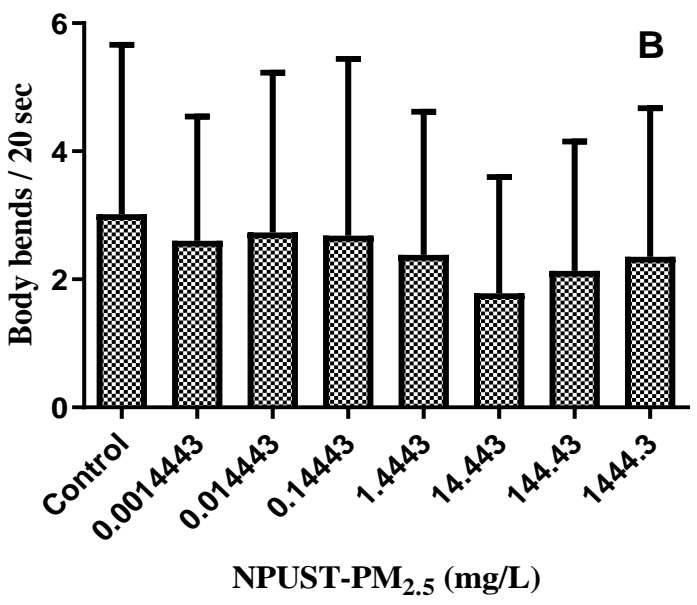

Body-bend frequency

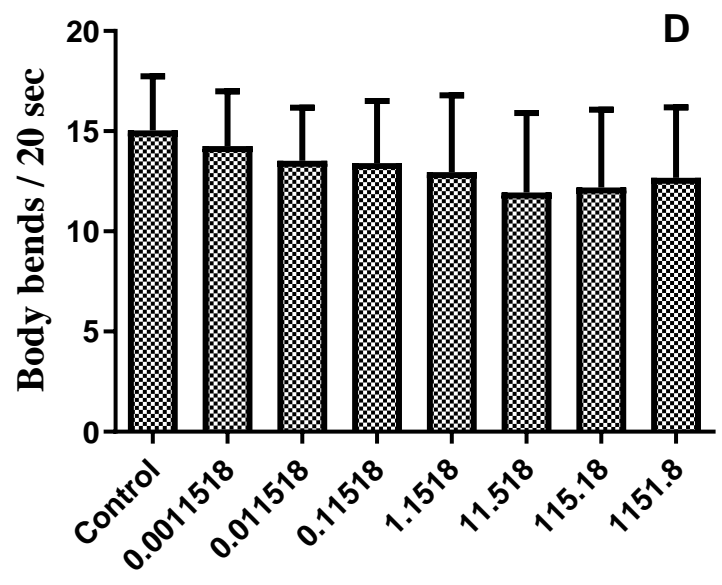

LJSH-PM $2.5(\mathrm{mg} / \mathrm{L})$

Fig. 5. Effects of $\mathrm{PM}_{2.5}$ from NPUST and (Bottom) LJHS on C. elegans locomotion and movement after 24-hour acute exposure. $\mathrm{A}$ and $\mathrm{C}$ for head thrashing and $\mathrm{B}$ and $\mathrm{D}$ for body bending.

\section{ACKNOWLEDGMENTS}

This study was supported by a grant from the Ministry of Science and Technology (MOST 106-2221-E-020-001-MY3). We acknowledge Mr. Ying-Jhih Syu from National Pingtung University of Science and Technology for assisting us to maintain and culture C. elegans. We also want to thank Miss Chia-Jung Yen from Kaohsiung Medical University for assisting us with the $C$. elegans experiments. We would also like to thank Dr. Chang-Shi Chen at National Cheng Kung University for his advice and help in attaining the C. elegans culture. The authors thanked the teachers and students in Linluo Junior High School for providing the air sampling site.

\section{DISCLAIMER}

The authors declare no conflicts of interest.

\section{REFERENCES}

Aoki, I. and Mori, I. (2015). Molecular biology of thermosensory transduction in C. elegans. Curr. Opin. Neurobiol. 34: 117-124.

Artifon, V., Zanardi-Lamardo, E. and Fillmann, G. (2019). Aquatic organic matter: Classification and interaction with organic microcontaminants. Sci. Total Environ. 649: 1620-1635.

Brenner, S. (1974). The genetics of Caenorhabditis elegans. Genetics 77: 71-94.

Brook, R.D. and Rajagopalan, S. (2010). Particulate matter air pollution and atherosclerosis. Curr. Atheroscler Rep. 12: 291-300.

Chao, H.R., Que, D.E., Gou, Y.Y., Chuang, C.Y., Chang, T.Y. and Hsu, Y.C. (2016). Indoor and outdoor concentrations of polybrominated diphenyl ethers on respirable particulate in central and southern Taiwan. Aerosol Air Qual. Res. 16: 3187-3197.

Chao, H.R., Hsu, J.W., Ku, H.Y., Wang, S.L., Huang, H.B., Liou, S.H. and Tsou, T.C. (2018). Inflammatory response and $\mathrm{PM}_{2.5}$ exposure of urban traffic conductors. Aerosol Air Qual. Res. 18: 2633-2642.

Clavijo, A., Kronberg, M.F., Rossen, A., Moya, A., Calvo, 
D., Salatino, S.E., Pagano, E.A., Morábito, J.A. and Munarriz, E.R. (2016). The nematode Caenorhabditis elegans as an integrated toxicological tool to assess water quality and pollution. Sci. Total Environ. 569570: 252-261.

Dodla, V.B.R., Gubbala, C.S. and Desamsetti, S. (2017). Atmospheric dispersion of $\mathrm{PM}_{2.5}$ precursor gases from two major thermal power plants in Andhra Pradesh, India. Aerosol Air Qual. Res. 17: 381-393.

El-Hajj, Z.W. and Newman, E.B. (2015). An Escherichia coli mutant that makes exceptionally long cells. $J$. Bacteriol. 197: 1507-1514.

Feng, S., Gao, D., Liao, F., Zhou, F. and Wang, X. (2016). The health effects of ambient $\mathrm{PM}_{2.5}$ and potential mechanisms. Ecotoxicol. Environ. Saf. 128: 67-74.

Fu, P., Guo, X., Cheung, F.M.H. and Yung, K.K.L. (2019). The association between $\mathrm{PM}_{2.5}$ exposure and neurological disorders: A systematic review and meta-analysis. Sci. Total Environ. 655: 1240-1248.

Gibson, M.D., Kundu, S. and Satish, M. (2013). Dispersion model evaluation of $\mathrm{PM}_{2.5}, \mathrm{NO}_{\mathrm{x}}$ and $\mathrm{SO}_{2}$ from point and major line sources in Nova Scotia, Canada using AERMOD Gaussian plume air dispersion model. Atmos. Pollut. Res. 4: 157-167.

Gonzalez-Moragas, L., Roig, A. and Laromaine, A. (2015). C. elegans as a tool for in vivo nanoparticle assessment. Adv. Colloid Interface Sci. 219: 10-26.

Hopke, P.K., Harrison, R.M., de Leeuw, F. and Querol, X. (2018). Chapter 1 - Current state of particulate air quality. In Non-exhaust emissions, Amato, F. (Ed.), Academic Press, pp. 1-19.

Hu, C.Y., Fang, Y., Li, F.L., Dong, B., Hua, X.G., Jiang, W., Zhang, H., Lyu, Y. and Zhang, X.J. (2019). Association between ambient air pollution and Parkinson's disease: Systematic review and meta-analysis. Environ. Res. 168: 448-459.

Hu, Y., Wang, L.S., Li, Y., Li, Q.H., Li, C.L., Chen, J.M., Weng, D. and Li, H.P. (2017). Effects of particulate matter from straw burning on lung fibrosis in mice. Environ. Toxicol. Pharmacol. 56: 249-258.

Huang, C.C., Chen, B.Y., Pan, S.C., Ho, Y.L. and Guo, Y.L. (2019). Prenatal exposure to $\mathrm{PM}_{2.5}$ and congenital heart diseases in Taiwan. Sci. Total Environ. 655: 880886.

Jun, X., Jin, G., Fu, C., Jinxuan, Z., Xuelin, L., Jiaxin, H., Shuaihua, Q., Anqi, S., Jianzhou, C., Lian, Z., Xiwen, Z., Baoli, Z. and Biao, X. (2018). PM $_{2.5}$ promotes abdominal aortic aneurysm formation in angiotensin II-infused apoe-/- mice. Biomed. Pharmacother. 104: 550-557.

Kaletta, T. and Hengartner, M.O. (2006). Finding function in novel targets: C. elegans as a model organism. Nat. Rev. Drug Discovery 5: 387-399.

Karimi, B., shokrinezhad, B. and Samadi, S. (2019). Mortality and hospitalizations due to cardiovascular and respiratory diseases associated with air pollution in Iran: A systematic review and meta-analysis. Atmos. Environ. 198: 438-447.

Khan, F., Jain, S. and Oloketuyi, S.F. (2018). Bacteria and bacterial products: Foe and friends to Caenorhabditis elegans. Microbiol. Res. 215: 102-113.

Kiesewetter, G., Schoepp, W., Heyes, C. and Amann, M. (2015). Modelling $\mathrm{PM}_{2.5}$ impact indicators in Europe: Health effects and legal compliance. Environ. Modell. Software 74: 201-211.

Kim, T.Y., Ho Kim, H., Yi, S.M., Cheong, J.P. and Heo, J. (2018). Short-term effects of ambient $\mathrm{PM}_{2.5}$ and $\mathrm{PM}_{2.5-10}$ on mortality in major cities of Korea. Aerosol Air Qual. Res. 18: 1853-1862.

Lee, Y.Y., Wang, L.C., Zhu, J., Wu, J.L. and Lee, K.L. (2018) Atmospheric $\mathrm{PM}_{2.5}$ and polychlorinated dibenzo$p$-dioxins and dibenzofurans in Taiwan. Aerosol Air Qual. Res. 18: 762-779.

Leung, M.C.K., Williams, P.L., Benedetto, A., Au, C., Helmcke, K.J., Aschner, M. and Meyer, J.N. (2008). Caenorhabditis elegans: An emerging model in biomedical and environmental toxicology. Toxicol. Sci. 106: 5-28.

Li, M., Hu, M., Guo, Q., Tan, T., Du, B., Huang, X., He, L., Song Guo, S., Wang, W., Fan, Y. and Xu, D. (2018a). Seasonal source apportionment of $\mathrm{PM}_{2.5}$ in Ningbo, a coastal city in southeast China. Aerosol Air Qual. Res. 18: 2741-2752.

Li, T., Zhang, Y., Wang, J., Xu, D., Yin, Z., Chen, H., Lv, Y., Luo, J., Zeng, Y., Liu, Y., Kinney, P.L. and Shi, X. (2018b). All-cause mortality risk associated with longterm exposure to ambient $\mathrm{PM}_{2.5}$ in China: A cohort study. Lancet Public Health 3: e470-e477.

Lin, Z., Niu, Y., Chen, R., Xu, W., Li, H., Liu, C., Cai, J., Zhao, Z., Kan, H. and Qiao, L. (2017). Fine particulate matter constituents and blood pressure in patients with chronic obstructive pulmonary disease: A panel study in Shanghai, China. Environ. Res. 159: 291-296.

Lo, W.C., Shie, R.H., Chan, C.C. and Lin, H.H. (2017). Burden of disease attributable to ambient fine particulate matter exposure in Taiwan. J. Formos. Med. Assoc. 116: 32-40.

Maciejczyk, P., Jin, L., Hwang, J.S., Guo, X., Zhong, M., Thurston, G., Qu, Q., Zhang, J., Sun, Q. and Chen, L.C. (2018). Association of cardiovascular responses in mice with source-apportioned $\mathrm{PM}_{2.5}$ air pollution in Beijing. Aerosol Air Qual. Res. 18: 1839-1852.

Mari, M., Sánchez-Soberón, F., Audí-Miró, C., van Drooge, B.L., Soler, A., Grimalt, J.O. and Schuhmacher, M. (2016). Source apportionment of inorganic and organic PM in the ambient air around a cement plant: Assessment of complementary tools. Aerosol Air Qual. Res. 16: 3230-3242.

Martens, D.S., Cox, B., Janssen, B.G., Clemente, D.B.P., Gasparrini, A., Vanpoucke, C., Lefebvre, W., Roels, H.A., Plusquin, M. and Nawrot, T.S. (2017). renatal air pollution and newborns' predisposition to accelerated biological aging. JAMA Pediatr. 171: 1160-1167.

Martins, N.R. and da Graça, GG. (2018). Impact of $\mathrm{PM}_{2.5}$ in indoor urban environments: A review. Sustainable Cities Soc. 42: 259-275.

Miri, M., Nazarzadeh, M., Alahabadi, A., Ehrampoush, M.H., Rad, A., Lotfi, M.H., Sheikhha, M.H., Sakhvidi, M.J.Z., Nawrot, T.S. and Dadvand, P. (2019). Air pollution and telomere length in adults: A systematic 
review and meta-analysis of observational studies. Environ. Pollut. 244: 636-647.

Mohammadyan, M., Alizadeh-Larimi, A., Etemadinejad, S., Latif, M.T., Heibati, B., Yetilmezsoy, K., Abdul-Wahab, S.A. and Dadvand, P. (2017). Particulate air pollution at schools: Indoor-outdoor relationship and determinants of indoor concentrations. Aerosol Air Qual. Res. 17: 857-864.

Moon, J., Kwak, J.I. and An, Y.J. (2019). The effects of silver nanomaterial shape and size on toxicity to Caenorhabditis elegans in soil media. Chemosphere 215: 50-56.

Owoade, K.O., Hopke, P.K., Olise, F.S., Ogundele, L.T., Fawole, O.G., Olaniyi, B.H., Jegede, O.O., Ayoola, M.A. and Bashiru, M.I. (2015). Chemical compositions and source identification of particulate matter $\left(\mathrm{PM}_{2.5}\right.$ and $\mathrm{PM}_{2.5-10}$ ) from a scrap iron and steel smelting industry along the Ife-Ibadan highway, Nigeria. Atmos. Pollut. Res. 6: 107-119.

Pandey, P., Patel, D.K., Khan, A.H., Barman, S.C., Murthy, R.C. and Kisku, G.C. (2013). Temporal distribution of fine particulates $\left(\mathrm{PM}_{2.5}, \mathrm{PM}_{10}\right)$, potentially toxic metals, PAHs and Metal-bound carcinogenic risk in the population of Lucknow City, India. J. Environ. Sci. Health., Part A 487: 730-745.

Pinkerton, K.E., Green, F.H.Y., Saiki, C., Vallyathan, V., Plopper, C.G., Gopal, V., Hung, D., Bahne, E.B., Lin, S.S., Ménache, M.G. and Schenker, M.B. (2000). Distribution of particulate matter and tissue remodeling in the human lung. Environ. Health Perspect. 108: 1063-1069.

Polezer, G., Tadano, Y.S., Siqueira, H.V., Godoi, A.F.L., Yamamoto, C.I., de André, P.A., Pauliquevis, T., Andrade, M.d.F., Oliveira, A., Saldiva, P.H.N., Taylor, P.E. and Godoi, R.H.M. (2018). Assessing the impact of $\mathrm{PM}_{2.5}$ on respiratory disease using artificial neural networks. Environ. Pollut. 235: 394-403.

Rai, N., Sjöberg, V., Forsberg, G., Karlsson, S., Olsson, P.E. and Jass, J. (2019). Metal contaminated soil leachates from an art glass factory elicit stress response, alter fatty acid metabolism and reduce lifespan in Caenorhabditis elegans. Sci. Total Environ. 651: 2218-2227.

Salako, G.O., Hopke, P.K., Cohen, D.D., Begum, B.A., Biswas, S.K., Pandit, G.G., Chung, Y.S., Rahman, S.A., Hamzah, M.S., Davy, P., Markwitz, A., Shagjjamba, D., Lodoysamba, S., Wimolwattanapun, W. and Bunprapob, S. (2012). Exploring the variation between EC and BC in a variety of locations. Aerosol Air Qual. Res. 12: 1-7.

Seneviratne, S., Handagiripathira, L., Sanjeevani, S., Madusha, D., Waduge, V.A.A., Attanayake, T., Bandara, D. and Hopke, P.K. (2017). Identification of sources of fine particulate matter in Kandy, Sri Lanka. Aerosol Air Qual. Res. 17: 476-484.

Sun, L., Lin, Z., Liao, K., Xi, Z. and Wang, D. (2015). Adverse effects of coal combustion related fine particulate matter $\left(\mathrm{PM}_{2.5}\right)$ on nematode Caenorhabditis elegans. Sci. Total Environ. 512-513: 251-260.

Sun, L., Wu, Q., Liao, K., Yu, P., Cui, Q., Rui, Q. and Wang, D. (2016). Contribution of heavy metals to toxicity of coal combustion related fine particulate matter $\left(\mathrm{PM}_{2.5}\right)$ in Caenorhabditis elegans with wild-type or susceptible genetic background. Chemosphere 144: 2392-2400.

Wang, M., Nie, Y., Liu, Y., Dai, H., Wang, J., Si, B., Yang, Z., Cheng, L., Liu, Y., Chen, S. and Xu, A. (2019). Transgenerational Effects of Diesel Particulate Matter on Caenorhabditis Elegans through Maternal and Multigenerational Exposure. Ecotoxicol. Environ. Saf. 170: 635-643.

Wang, S., Chu, Z., Zhang, K. and Miao, G. (2018). Cadmium-induced serotonergic neuron and reproduction damages conferred lethality in the nematode Caenorhabditis elegans. Chemosphere 213: 11-18.

Wang, X., Chen, M., Zhong, M., Hu, Z., Qiu, L., Rajagopalan, S., Fossett, N.G., Chen, L.C. and Ying, Z. (2017). Exposure to concentrated ambient $\mathrm{PM}_{2.5}$ shortens lifespan and induces inflammation-associated signaling and oxidative stress in drosophila. Toxicol Sci. 156: 199-207.

World Health Organization (WHO) (2016). Dioxins and their effects on human health. https://www.who.int/en/n ews-room/fact-sheets/detail/dioxins-and-their-effects-on -human-health.

Xu, M.X., Ge, C.X., Qin, Y.T., Gu, T.T., Lou, D.S., Li, Q., $\mathrm{Hu}$, L.F., Feng, J., Huang, P. and Tan, J. (2019). Prolonged $\mathrm{PM}_{2.5}$ exposure elevates risk of oxidative stress-driven nonalcoholic fatty liver disease by triggering increase of dyslipidemia. Free Radical Biol. Med. 130: 542-556.

Yan, F., Zhu, F., Wang, Q. and Xiong, Y. (2016). Preliminary study of $\mathrm{PM}_{2.5}$ formation during municipal solid waste incineration. Procedia Environ. Sci. 31: 475-481.

Yang, B. and Xiao, C. (2018). $\mathrm{PM}_{2.5}$ exposure significantly improves the exacerbation of A549 tumor-bearing CB17SCID mice. Environ. Toxicol. Pharmacol. 60: 169-175.

Yang, Z., Xue, K.S., Sun, X., Williams, P.L., Wang, J.S. and Tang, L. (2018). Toxicogenomic responses to zearalenone in Caenorhabditis elegans reveal possible molecular mechanisms of reproductive toxicity. Food Chem. Toxicol. 122: 49-58.

Zhao, C., Niu, M., Song, S., Li, J., Su, Z., Wang, Y., Gao, Q. and Wang, H. (2019). Serum metabolomics analysis of mice that received repeated airway exposure to a water-soluble $\mathrm{PM}_{2.5}$ extract. Ecotoxicol. Environ. Saf. 168: 102-109.

Zhao, R., Cui, K., Wang, W., Wang, L.C. and Yan, P. (2018). Atmospheric $\mathrm{PM}_{2.5}$ and total PCDD/Fs- $\mathrm{WHO}_{2005}$ TEQ level: A case of Handan and Kaifeng cities, China. Aerosol Air Qual. Res. 18: 994-1007.

Zhao, Y., Lin, Z., Jia, R., Li, G., Xi, Z. and Wang, D. (2014). Transgenerational effects of traffic-related fine particulate matter $\left(\mathrm{PM}_{2.5}\right)$ on nematode Caenorhabditis elegans. J. Hazard. Mater. 274: 106-114.

Zhou, Z., Shao, T., Qin, M., Miao, X., Chang, Y., Sheng, W., Wu, F. and Yu, Y. (2018). The effects of autophagy on vascular endothelial cells induced by airborne $\mathrm{PM}_{2.5}$. J. Environ. Sci. 66: 182-187. 
Zuo, Y.T., Hu, Y., Lu, W.W., Cao, J.J., Wang, F., Han, X., Lu, W.Q. and Liu, A.L. (2017). Toxicity of 2,6dichloro-1,4-benzoquinone and five regulated drinking

Received for review, March 21, 2019

Revised, April 23, 2019 water disinfection by-products for the Caenorhabditis Accepted, April 24, 2019 elegans nematode. J. Hazard. Mater. 321: 456-463. 